\title{
Maximising inductive power transmission using a novel analytical coil design approach.
}

\author{
HEIDARIAN, M., BURGESS, S.J., PRABHU, R. and FOUGH, N.
}

(c) 2019 IEEE. Personal use of this material is permitted. Permission from IEEE must be obtained for all other uses, in any current or future media, including reprinting/republishing this material for advertising or promotional purposes, creating new collective works, for resale or redistribution to servers or lists, or reuse of any copyrighted component of this work in other works. 


\title{
Maximising Inductive Power Transmission using a Novel Analytical Coil Design Approach
}

\author{
Maryam Heidarian, Samuel J. Burgess, Radhakrishna Prabhu, Nazila Fough \\ School of Engineering \\ Robert Gordon University \\ Aberdeen, United Kingdom \\ M.Heidarian@rgu.ac.uk, S.J.Burgess@rgu.ac.uk
}

\begin{abstract}
Maximising power transfer efficiency (PTE) in resonant inductive power transfer (IPT) systems requires strong coupling between transmitter and receiver coils. In applications where system constraints yield a weak inductive link (e.g.: significant distance between coils.) or there is a requirement for a specific power level, then geometrically optimising the coils can enhance inductive linkage. To achieve this, a novel coil design method has been presented which provides maximum efficiency for both strongly- and loosely-coupled inductive links. A parameter (i.e.: Strong Coupling Factor.) has been introduced to assist the design procedure. Discussed results from a practical 1.06 $\mathrm{MHz}$ inductive link, developed using the proposed design method, show that with proper selection of strong coupling factor (e.g.: $\mathbf{C}=220$.) the designed coil geometry can provide maximum PTE of $86 \%$, which is in close correlation $(\approx 3 \%)$ with theoretical analysis using MATLAB.
\end{abstract}

Index Terms-Wireless power transfer (WPT), inductive power transfer (IPT), resonant coupling, electromagnetic induction, near-field communication.

\section{INTRODUCTION}

Resonant inductive coupling is a well established method of wireless power transfer (WPT). The operation principal of this signal (i.e.: power or data.) transfer technique is based on resonant coupling of a magnetic field between a primary and a secondary coil. This wireless coupling operation makes IPT ideal for diverse short-range and mid-range WPT applications, such as: wireless battery charging, implantable biomedical devices, radio frequency identification (RFID) sensors, wireless microelectromechanical systems (MEMS), etc.

A major WPT challenge is achieving maximum PTE [1]. PTE is affected by: coil size (e.g.: diameter, length, number of turns, etc.), transmission medium distance and attenuation level, plus terminating circuitry of both receiver and transmitter.

This paper proposes an optimum coil design procedure based on "Strong Coupling Factor", which in turn allows manipulation of coil ohmic resistance to produce a set of physical values for construction of coils in an inductive link. The novel approach can be shown to deliver a theoretical maximum PTE; which has been verified by experimental testing. The technique lends itself well to IPT applications where the physical size and shape of transceiver is limited.

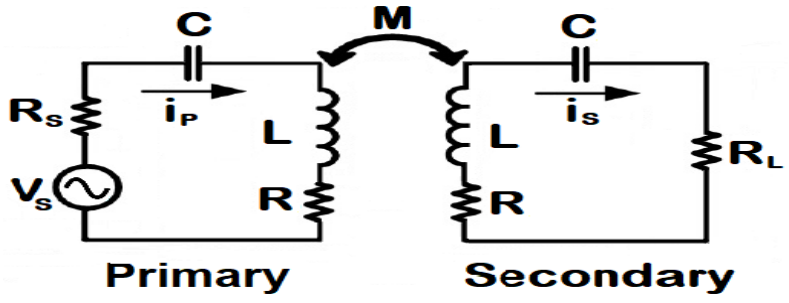

Fig. 1: Circuit model.

\section{TheoretiCAL AnAlysis}

Operating a transceiver inductive link at magnetic resonance maximises the linking action. A simplified "lumped resonant IPT system", based upon [2], is shown in Fig. 1. In this model both primary and secondary coils are considered identical. The $L C$-tanks are tuned to an operating resonant frequency $f_{o}\left(\omega_{o}=2 \pi f_{o}=1 / \sqrt{L C}\right)[1]-[2]$, where $L$ is the inductance value of the primary and secondary coils, and capacitor $C$ is their respective resonance pair. The primary side is considered as an ac voltage source, $V_{s}$, with an impedance, $R_{s}$. The secondary side $R_{L}$ represents a load (dc or ac). $R$ is the winding ohmic resistance of the primary and the secondary coils. $M$ is the mutual inductance between coils.

\section{A. Maximum Power Transfer Efficiency Formulation}

At resonance the power transfer efficiency, $\eta$, of the transceiver system in Fig. 1 can be expressed as:

$$
\eta=\frac{R_{L}\left|\mathbf{I}_{\mathbf{s}}\right|^{2}}{V_{s}\left|\mathbf{I}_{\mathbf{p}}\right|}=\frac{K^{2} Q_{p} Q_{s}}{1+K^{2} Q_{p} Q_{s}}\left(1-\frac{Q_{s}}{Q_{i}}\right)
$$

where $K$ is the coupling coefficient between the coils, $K=$ $M / L$. The primary and secondary loaded quality factors are included as: $Q_{p}=\omega_{o} L /\left(R+R_{s}\right)$ and $Q_{s}=\omega_{o} L /\left(R+R_{L}\right)$. $Q_{i}$ is the intrinsic $Q$ factor of the coil, $Q_{i}=\omega_{o} L / R$. From (1) a condition which guarantees maximum PTE (i.e.: $\eta=1$.) can be expressed as:

$$
\left\{\begin{array}{l}
Q_{i}-Q_{s} \equiv Q_{i} \\
K^{2} Q_{p} Q_{s} \equiv 1+K^{2} Q_{p} Q_{s} .
\end{array}\right.
$$

These require both the following inequalities to be satisfied:

$$
\left\{\begin{array}{c}
Q_{i} \gg Q_{s} \\
K^{2} Q_{p} Q_{s} \gg 1 .
\end{array}\right.
$$


With proper coil wire selection inequality (4) is met, i.e.: a low ohmic resistance yields large $Q_{i}$. Inequality (5) is the electrical representative of "Strong Coupling Conduction" [1], [3]. A strong coupling link between transmitter and receiver coils requires -

1) An inductive link with high coupling coefficient " $K$ ": The transmission medium gap and coil mutual orientation set $K$ between 0 and 1 (i.e.: $0 \leq K \leq 1$.).

2) A transceiver system with low terminating impedances $\left(R_{s} \& R_{L}\right)$ : The optimum low values for $R_{s}$ and $R_{L}$ will vary depending on system power level and application.

3) Transmitter and receiver coils with high self-inductance and small ohmic resistance: Equation (6) expresses coil (i.e.: coreless solenoid shown in Fig. 2.) self-inductance as [4]:

$$
L=\frac{\mu_{o} \pi N^{2} r_{c}^{2}}{\sqrt{4 r_{c}^{2}+l_{c}^{2}}}
$$

in which $\mu_{o}$ is free space permeability, $N$ is number of turns, $r_{c}$ is coil radius and $l_{c}$ is coil length (where $l_{c} \gg r_{c}$ ). Also, the coil ohmic resistance with inclusion of the skin effect on winding wire, based on [3], can be calculated as:

$$
R=\frac{N r_{c} \sqrt{2 \omega_{o} \mu \rho}}{d_{w}}
$$

where $\mu$ and $\rho$ are permeability and resistivity of winding wire material, and $d_{w}$ is wire diameter. From (6), increasing $N$ and $r_{c}$ improves coil self-inductance. However, increasing these values has the detrimental effect of raising the coil ohmic resistance.

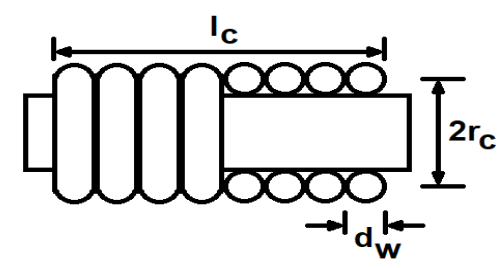

Fig. 2: Solenoid structure.

The challenge is to design a geometrically optimised coil with maximised self-inductance, while concurrently exhibiting a low ohmic resistance.

\section{B. Optimum Coil Design for Strong Coupling}

Finding an optimal coil geometry to maximise PTE for a certain $R_{s}$ and $R_{L}$ requires exploiting the coil self-inductance to yield an ohmic resistance that provides strong coupling between primary and secondary coils.

To ease the calculations, the first design step in selecting a suitable coil ohmic resistance is to introduce a constant parameter $C(C \gg 1)$ to represent the "Strong Coupling Factor". Thus (5) can be restated as:

$$
K^{2} Q_{p} Q_{s}=C \Rightarrow K^{2} \omega_{o}^{2} L^{2}=C\left(R+R_{s}\right)\left(R+R_{L}\right) .
$$

As the coil self-inductance needs to be maximised we solve (8) for $R$ to yield the ohmic resistance which maximises PTE for a given IPT system.
The coil self-inductance, (6), is a factor of three variables; $N, r_{c}$ and $l_{c}$. Replacing $N$ in (6) with its equivalent from (7) permits $L$ to be restated based on $r_{c}$ as:

$$
L=\frac{\mu_{o} \pi R^{2} d_{w}^{2} r_{c}}{\sqrt{\left(2 \omega_{o} \mu \rho\right)\left(R^{2} d_{w}^{4}+8 r_{c}^{4} \omega_{o} \mu \rho\right)}} .
$$

In a tightly wound coil $l_{c}=N d_{w}$. Differentiating (9) with respect to $r_{c}$ provides an $r_{c}$ which maximizes $L$ :

$$
r_{c}=\sqrt[4]{\frac{R^{2} d_{w}^{4}}{8 \omega_{o} \mu \rho}}=\frac{l_{c}}{2} \Rightarrow L_{\max }=\sqrt[4]{\frac{\mu_{o}^{4} \pi^{4} d_{w}^{4} R^{6}}{128 \omega_{o}^{3} \mu^{3} \rho^{3}}} .
$$

Substituting the coil self-inductance with its maximum value in (8) yields:

$$
\left(\frac{\left(K \omega_{o} \mu_{o} \pi d_{w}\right)^{2}}{4 C\left(2 \omega_{o} \mu \rho\right)^{3 / 2}}\right) R^{3}-R^{2}-\left(R_{s}+R_{L}\right) R-R_{s} R_{L}=0 .
$$

Solving (11) for $R$ determines the ohmic resistance. The novelty of this design approach is that $R$ has been established based on the coil self-inductance thus guaranteeing a maximised PTE. With $R$ established, $r_{c}, N$ and $l_{c}$ can be calculated from (6) and (7).

\section{Design EXAMPLE \& EXPERIMENTAL Results}

Using our novel design approach an IPT system was constructed at an operational test frequency of $f_{o}=1.06 \mathrm{MHz}$ as shown in Fig. 3. The source and load resistances, chosen to complement the design method, were $R_{s}=3 \Omega$ and $R_{L}=3.5$ $\Omega$ respectively. For an arbitrary $C$ value of $220(C=220)$ the calculated ohmic resistance value from (11) is $R=152.3 \mathrm{~m} \Omega$. For this $R$ value $r_{c}=9.5 \mathrm{~mm}, N=24$ and $l_{c}=19.0 \mathrm{~mm}$. The coil winding conductor used is $0.8 \mathrm{~mm}$ (dia) copper wire and the coupling coefficient has been considered as $K=1$ (i.e.: a strong inductive link.).

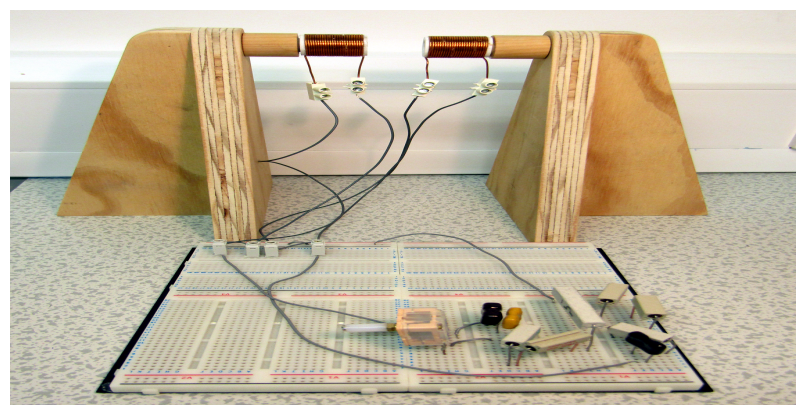

Fig. 3: Experimental PTE test setup.

For the physical circuit parameters developed by our design method, the maximum calculated PTE, using MATLAB, is $95.4 \%$ as shown in Fig. 4. With a fall in coupling coefficient, over a nominal range of $0.1 \leq K \leq 1$, the link becomes more loosely-coupled but the maximum PTE becomes apparent as being around the designed coil $r_{c}$ value.

To prove the concept, for a practically optimum $K$ value of 0.215 ( $K=0.215)$, Fig. 5 shows test results on three $1.06 \mathrm{MHz}$ IPT systems, each with different $r_{c}$ values. The experimental PTE value shows remarkable closeness to the 


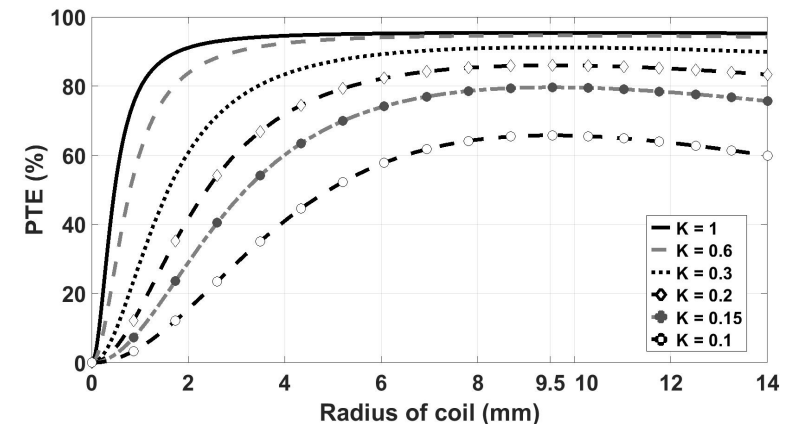

Fig. 4: Maximum PTE for different coupling coefficients $(K)$.

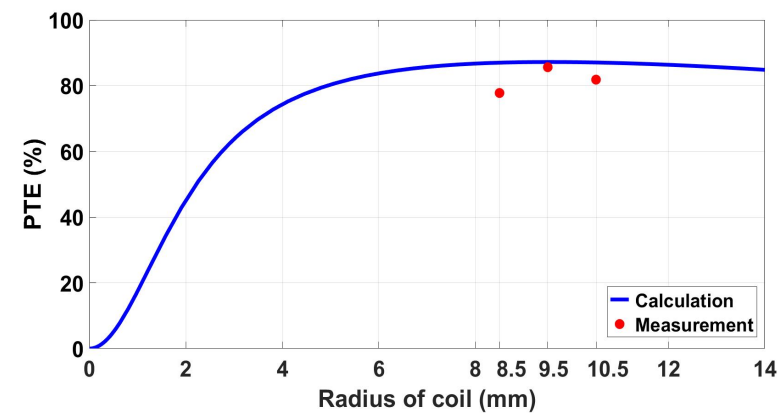

Fig. 5: Calculated \& measured efficiency for $K=0.215$.

analytical prediction: the measured peak efficiency is $86 \%$ while calculations, based on physical coil properties, result in $89 \%$ efficiency. Tests on physical geometries either side of $r_{c}=9.5 \mathrm{~mm}$ show falling PTE values, thus validating the design method proposed.

Maximum PTE highly depends on coupling coefficient, which is a function of relative distance between the primary and secondary coils. Fig. 6 demonstrates calculated and measured PTE over a transmission distance, $d_{t}$, ranging from 9.5 $\mathrm{mm}$ to $49.5 \mathrm{~mm}$ (i.e.: $K=0.215$ to $K=0.013$.). The calculations show that with an increase in distance between primary and secondary coils the system's PTE falls with a predictable slope. One can consider the coils' distance increment ratio as $\alpha$; hence, it can be seen that the PTE attenuation slope depends on how far the coils are apart from each other - attenuation is an inverse factor of $\alpha$. For transmission distances less than coil radius (i.e.: $d_{t} \leq r_{c}$.), PTE falls with a ratio of $\alpha^{-1}$. In the case where the distance between the coils is $r_{c}<d_{t} \leq 2 r_{c}$; the PTE reduces with a slope of $\alpha^{-2}$. For $d_{t}$ higher than $2 r_{c}$ (i.e.: $d_{t}>2 r_{c}$.) the PTE will reduce with a factor of $\alpha^{-3}$, this agrees well with signal attenuation observations in near-field communications [3]. The measured values follow the calculations closely.

Another important factor affecting PTE is the terminating impedances of the IPT system (i.e.: $R_{s} \& R_{L}$.), since the level of power dissipated in the source impedance, $R_{s}$, determines what remaining power is left for the inductive link. The PTE of the designed IPT system, with $K=0.215$, for different terminating impedances is presented in Fig. 7(a). As expected,

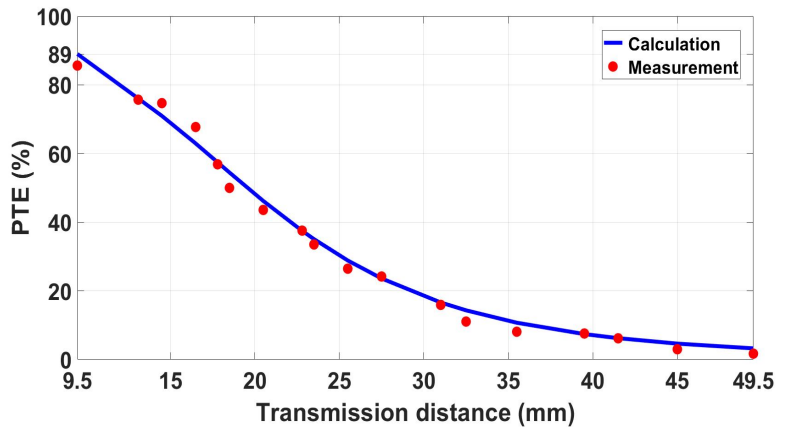

Fig. 6: PTE versus distance variations.

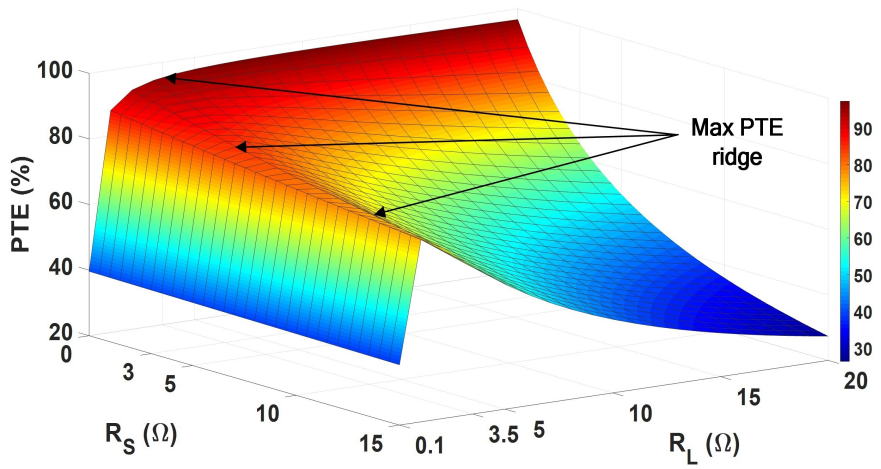

(a)

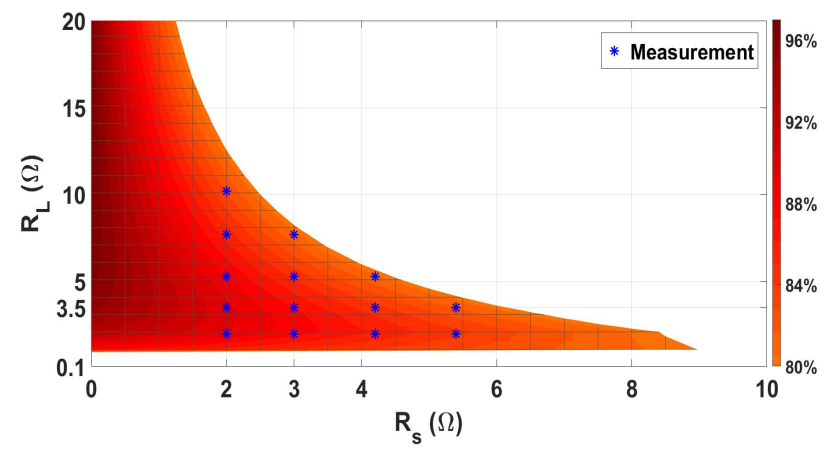

(b)

Fig. 7: (a) PTE versus terminating impedances variations. (b) Terminating impedances yielding calculated \& measured PTE higher than $80 \%$.

with an increase in $R_{s}$, PTE will reduce regardless of $R_{L}$. The 3D curve demonstrates that for each source impedance maximum PTE occurs at a single optimum load value. Using small $R_{s}$ values (i.e.: $R_{s} \leq 3 \Omega$.) results in optimum $R_{L}$ values $\geq 3.5 \Omega$. As $R_{s}$ increases, optimum $R_{L}$ must be reduced to lower values $(\approx 1 \Omega)$ : given by the "Max PTE ridge" in the curve. This results in careful selection of $R_{s}$ when using a high $R_{L}$ value to achieve a certain power level in the secondary side. It can also be observed from Fig. 7(a) that our coil design method can accommodate variations in terminating impedances. In order to visualise this relationship the 3D plot in Fig. 7(a) has been transformed into a 2D plot in Fig. 7(b). 


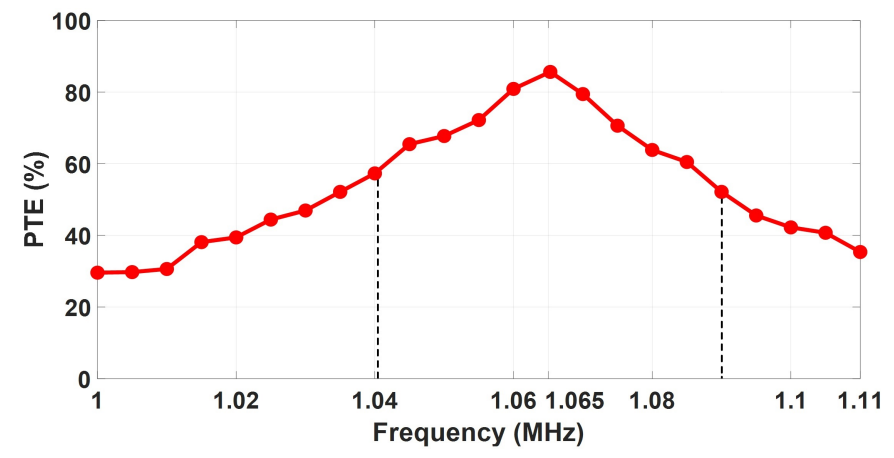

Fig. 8: PTE versus operating frequency variations.

In this figure the shaded area represents the corresponding $R_{s}$ and $R_{L}$ values that give a PTE $\geq 80 \%$. For example, with $R_{s}=3 \Omega$ the value of $R_{L}$ can range between $1 \Omega$ to $8 \Omega$ and PTE remains $\geq 80 \%$. The PTE of several practical impedance combinations in this range have been physically measured to validate the calculations.

In a resonant-based IPT system practical measurements of maximum PTE requires accurate resonance frequency tuning of both primary and secondary circuits. Fig. 8 demonstrates if the resonance frequency is detuned $\pm 2.5 \%$, the measured maximum PTE drops by $\approx 39 \%$. The centre frequency of the measurement setup is $1.065 \mathrm{MHz}$.

\section{CONCLUSION}

A novel design approach to maximise PTE through coil geometry optimisation has been presented. By introducing the concept of strong coupling factor, $C$, the proposed coil design technique provides a high level of PTE for both strongly- and loosely-coupled IPT inductive links. Simulated and practical measurements produced optimised results within $3 \%$ difference; validating the design approach. Also, it was shown that the presented method displays a high predictability with variations in coupling coefficient $(K)$, terminating impedances $\left(R_{s} \& R_{L}\right)$ and resonance frequency $\left(f_{o}\right)$.

\section{ACKNOWLEDGMENT}

The authors would like to thank Dr. C. McCullagh for facilitating practical measurements and Mr. D. Smith for his technical assistance.

\section{REFERENCES}

[1] M. Kiani and M. Ghovanloo, "The circuit theory behind coupled-mode magnetic resonance-based wireless power transmission", IEEE Trans. Circuits Syst. I, Reg. Papers, vol. 59, no. 9, pp. 2065-2074, 2012.

[2] W. Zhong, and S. Y. R. Hui, "Maximum energy efficiency operation of series-series resonant wireless power transfer systems using on-off keying modulation", IEEE Trans. on Power Electronics, vol. 33, no. 4, pp. 35953603,2018

[3] A. Kurs, A. Karalis, R. Moffatt, J. D. Joannopoulos, P. Fisher, and M. Soljačić, "Wireless power transfer via strongly coupled magnetic resonances", Sci. Exp., vol. 317, no. 5834, pp. 83-86, 2007.

[4] M. Sadiku, Elements of electromagnetics. Oxford university press, 2015. 